INSIGHT

Journal of Critical Dietetics

ISSN 1923-1237

Vol 5, Issue 2

Copyright 2021

Toronto, $\mathrm{ON}$

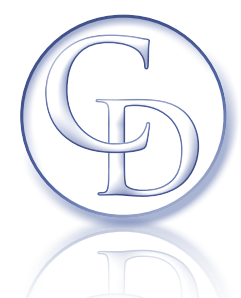

\title{
Call to Action to Improve Racial Diversity in Dietetics
}

\author{
Anisha Mahajan MPH, RD, CDE'; Ananya T. Banerjee PhD²; Maria Ricupero MHSc, RD, CDE; Amanda Beales MAN, RD, CDE; \\ Jane Lac MPH, RD, CDE; Fatim Ajwani RD; Avantika Mathur-Balendra MSc²; Twinkle Patel RD; Vanita Pais RD, CDE \\ I Department of Human Health and Nutritional Sciences, University of Guelph, Guelph, Ontario \\ 2 Dalla Lana School of Public Health, University of Toronto, Toronto, Ontario \\ 3 Department of Endocrinology, Hospital for Sick Children, Toronto, Ontario \\ Correspondence to: \\ Anisha Mahajan (Doctoral student and a Registered Dietitian) \\ University of Guelph, 50 Stone Road East, Guelph, Ontario NIG 2WI \\ Email: anishamahajan29@gmail.com Phone: 416-888-2607 \\ Funding agencies: Not applicable / None
}

\section{Background}

A diverse dietitian workforce is needed to better meet the needs of the ever-increasing multicultural population in Canada where "more than one in five Canadians [are] foreign born" and "two in five children [have] an immigrant background" (Statistics Canada, 2017). To achieve this increased representation within dietetics, it must parallel the rich variety of Indigeneity, races and ethnicities in our country. Racial diversity in the dietetic (or any) workforce reduces discrimination and structural inequalities and equalizes components of power and privilege by lessening oppression (Brady \& Gingras, 2019). This allows all voices to be heard including many authors' of this paper who represent the lived realities of racialized communities and instills varying perspectives related to race and class. Studies have shown that a racially diverse workforce in healthcare provides a wide range of benefits for racialized patients. These include improved patient satisfaction and trust, better access to healthcare professionals, reduced language barriers and enhanced quality of care along with better health outcomes of patients (Smedley \& Mittman, 20ll).

The history of dietetics as a profession began with its initial housing in the field of Home Economics in the late nineteenth century, and included mostly women at the time (Brady, 2018). Thereafter, dietetics arose as an independent profession in the twentieth century (Brady, 2018). From these beginnings to now, the current professional membership remains predominantly white females belonging to the middle and upper class (Brady, 2018). By comparison, recently the demographic makeup of dietitians in the United States of America (USA) in June, 2020 was found to be $72.5 \%$ Caucasians and $85 \%$ female (Commission on Dietetic Registration [CDR], 2020). This information is based on self-reported data gathered and compiled online from the Commission on Dietetic Registration of the Academy of Nutrition and Dietetics. There is a lack of such demographic data in Canada and this serves as a barrier for any objective analysis. Moreover, many dietitians may not be a member of the national dietetic organization to have this information available in one repository. The Canadian Institute for Health Information $(\mathrm{ClHI})$ gathers data on health workforce and has information available on percentage of practicing dietitian (and sex information) by province (ClHI, 2020). Race-based data collection would assist in tracking changes over time in racial diversity amongst dietetic practitioners, undergraduate and graduate students, and dietetic interns entering the 
field in the future. Thus, barriers should be reviewed and opportunities sought to gather this information for the dietetics profession at large.

The aim of our paper is to urge dietetic practitioners, leaders and educators alike to steadily and efficiently improve racial diversity in their respective academic and clinical institutions. In our view, a predetermined percentage of Indigenous and Black people should be prioritized and empowered into dietetics; the dietetic education model should be reviewed and barriers to entry for racialized groups (into the field) should be identified. We advocate for purposeful recruitment strategies as these are needed to increase representation from Indigenous and Black groups. The rationale for focusing on these groups is important given their shared and distinct histories of resistance and oppression. To present day, these groups face disproportionate barriers in healthcare access and experience continued systemic discrimination (National Collaborating Centre for Indigenous Health [NCCIH], 20l2; Hall et al., 20I5). The Indigenous peoples continue to encounter multiple challenges including food insecurity, a higher prevalence of type 2 diabetes, cardiovascular disease and stroke, have increased concerns of mental health including suicide, anxiety and depression and severe challenges with health inequities $(\mathrm{NCClH}, 2012)$. Furthermore, there is research and history on the beginnings of nutritional inequities (starting 1940s) and the indication that "nutrition experiments" were conducted in the Indigenous communities (Mosby, 2013). Research also indicates that students from African American backgrounds are discouraged in the USA to enter dietetics when compared with students from other backgrounds (White, 2018). These students are often intimidated to enter dietetics as the African culture has differences in opinions on healthy eating and body image versus recommendations from healthcare professionals (White, 2018). Lastly, along with focusing on these underrepresented communities, we need to consider the barriers to entry for all racialized groups into dietetics. A recent Canadian study suggested that dietetic students from Caucasian backgrounds were 3.8 times more likely to become a dietitian compared to students from racialized groups (Riedegier et al., 2019). The primary concerns to becoming a dietitian for racialized students were applications for student loans and stress with the application process (Riedegier et al., 2019). We suggest that all these concerns need further examination in the Canadian context and urge for further research in this area of dietetics.

\section{Call to Action}

In the latter half of this paper, we provide recommendations in the hopes of challenging the status quo and inspire change, as now more than ever we need systems-level solutions to address the racial diversity gaps in our profession. We acknowledge that diversity in dietetics may also include age, sex, ability, class, size and sexual orientation, however the scope of this paper is focused on racial equality that may intersect with the above social identities. Given the significant gap in the Canadian dietetics' literature on racial diversity, we have drawn from research in various healthcare fields and geographical areas. The following reveals our collective recommendations that focus on the different areas within dietetics:

\section{Dietetic Organizations:}

- National and provincial regulatory bodies along with organizations enforcing dietetic competencies in Canada, should include improving racial diversity into their strategic plans. Moreover, ways to achieve these plans and goals should be outlined, since these organizations influence dietetic practice. The dietetic competencies for incoming dietetic interns should enlist specific and measurable short-and long-term learning objectives to increase understanding on racial inequities in Canada.

- Canadian universities and stand-alone dietetic programs as well as the national dietetic organization should advertise or fundraise for monetary assistance to provide financial support in the form of scholarships, bursaries and grants to attract and retain a diverse student pool (Burt et al., 2019). The Academy of Nutrition and Dietetics (USA) has set up a foundation for the aforementioned purpose. Leaders from university dietetic programs, industry organizations, dietetic practice and other dietetic organizations should come together and make a concerted effort to support more racialized dietetic students.

- Dietetic Organizations should hire external consultants through agencies such as the Canadian Centre for Diversity and Inclusion to complete impartial audits and gain further insights and solutions to improve diversity and inclusion for the students entering into the profession. However, these audits can understandably become costly for smaller organizations. Alternatively, monetary funds can be allocated for training leaders of the organizations and 
taskforces, committees and working groups can be set up within the dietetic organizations to increase focus of diversity, equity and inclusion. Furthermore, Canadian dietetic journals should continue to encourage and increase funding for research studies to promote and amplify these initiatives.

\section{Dietetic Practice:}

- Dietetic professionals must reflect on concerns regarding the lack of diversity within their institutions and within their own practice.

- Dietetic professionals are encouraged to learn and self-reflect on implicit and inherent racial biases. These are well-documented in research for healthcare professionals that suggests discrimination during healthcare access towards Black, Hispanic, Latino, Latina and darker skinned individuals (Hall et al., 2015). Implicit biases can have a considerable impact on patient-provider interactions such as patient-centeredness, patient-provider collaboration and patient respect towards healthcare providers (Hall et al., 2015). These are important learnings that dietetic professionals can draw on within their own practice.

- Dietitians should provide mentorship and activities to increase exposure and interest of racialized students towards dietetics (Burt et al., 2019). Mentorship can play a significant role by pairing racialized students with mentors based on mentees' comfort level and preference (Burt et al., 2019). The goal is to create a safe space for learners to engage.

\section{Dietetic Education and Recruitment:}

- Indigenous cultural safety courses should be integrated into undergraduate studies and dietetic intern competencies across Canada. "Cultural Safety", a concept developed by Indigenous Mãori nurse leaders in Aotearoa (New Zealand). The theory of cultural safety "extends the notion of transcultural [care], with its focus on understanding the culture and values of nutrition of different ethnoracial groups, to include power inequities, individual and institutional discrimination surrounding a history of colonialism" (Polaschek, 1998). Although, there is limited research conducted on this topic in Canada, a study by Huycke et al. (2017) surveyed a national sample of dietitians in the Dietitians of Canada Aboriginal Nutrition Network to review the importance of Aboriginal Cultural Competence in dietetic practice and dietitian competencies. Ninetyfive percent of the respondents $(n=42)$ in this study believed that dietitians and dietetic interns must be competent in Aboriginal health and culture (Huycke et al., 2017).

- At the present time, cultural sensitivity training, anti-racism and anti-oppression workshops are not provided to all students and practitioners in dietetics (albeit, these are being initiated in many educational and employment settings). Based on our work experiences, there is a need to make these offerings mandatory and ongoing for all undergraduate and graduate students, dietetic interns and dietitians. Additionally, this training should be incorporated into dietetic competencies as well as new dietitian orientation programs.

- Aside from the strong focus on the clinical manifestation of disease and nutrition interventions in undergraduate programs, the important impact and contribution of the social determinants of health must be introduced early and interspersed throughout undergraduate studies and dietetic internship programs. This would include addressing how low income, housing instability, mental health and gender diversity among other social determinants impact on an individuals' health outcomes (Government of Canada, 2019).

- Currently, many students are not aware of the option to apply to the field of dietetics after high school. A needs assessment by undergraduate university dietetic programs can assist to increase participation from racialized communities. Outreach programs are required to introduce undergraduate dietetic programs to high school students, counsellors, parents and teachers (Burt et al., 2019). These can also be promoted to attract increased Black and Indigenous populations into dietetics. Collaborations and partnerships with student-focused organizations that service racialized youth can also be considered to increase awareness of the dietetics field of practice.

- There is also a striking lack of representation of racial diversity in academia (i.e. graduate programs and university professors) along with a sparsity of literature focused on diversity in dietetics in Canada. Currently, to our knowledge, multiple barriers exist that prevent more Canadian dietitians from entering academic positions. A study based in the United Kingdom reviewed barriers faced by women, 
Black minority ethnic groups and students living with disability from entering the field of academia (Mattocks \& Briscoe-Palmer, 2016). Seven themes emerged from this research study including: lack of institutional support, limited finances and funding, low confidence and self-esteem, external responsibilities, less focus on health and well-being, concerns of future professional life and isolation, exclusion and disadvantage (Mattocks \& Briscoe-Palmer, 2016). Additional investigation in the Canadian context is required to investigate and address barriers for racialized students interested in entering academia.

\section{Research and Theoretical Framework:}

- Further research is required to explore Critical Race Theory as an effective framework for the field of dietetics, especially when investigating the structural inequalities for racialized communities of the dietetic profession. This theoretical framework has its origins in legal studies and strives for social justice by allowing examination of various levels of influences of race on racialized groups (White, 2013). Critical Race Theory was introduced in the 1970s and was established by legal scholars namely Derrick Bell and Alan Freeman (White, 2013). To our knowledge, this theory has previously only appeared in nutrition research in a paper on African American nutrition educators as a medium to speak to inequities in the dietetics profession (White, 2013). This theory is based on the following tenets: firstly, racism is present in all sections of our society; secondly, lived experiences of racialized minorities can be understood through storytelling and lastly, this theory operates on the assumption that the dominant members of society will encourage the underrepresented groups only when it is guided by their own self-interests (White, 2013).

- Due to limited research on diversity in dietetics in Canada, practitioners, leaders and educators can look to successful experiences and ideas from other disciplines, fields of study and countries for direction.

$>$ The field of nursing maintains a paid clinical preceptor model as a promising and sustainable solution. Applying a similar mentorship model to dietetics can provide an opportunity to not only hire dietitians from various backgrounds, but also allow dietitians to receive honoraria/compensation for mentoring students (White \& Beto, 2013). Also, the field of nursing incentivizes racialized students by providing scholarships that allow for ease of entry into the field (White \& Beto, 2013). In Ontario, the Ministry of Training, Colleges and Universities, provide $\$ 50.00$ per week per student for "preceptor recognition" and "capacity building" (Council of Ontario Universities, 2013). The preceptor involvement incentive is extended to many allied health professionals in rehabilitation sciences, however this does not apply to registered dietitians at present time (Council of Ontario Universities, 2013).

- The National Association of Social Work in the United States of America supports diversity and social justice and has supervised field experiences within the degree requirements (Burt et al., 2019). This association has been successful in attracting students from low socio-economic status backgrounds and racialized communities (Burt et al., 2019). As part of dietetic internships and practicum placements for students, field experiences that increase awareness on racial and social justice issues should be incorporated as these currently do not exist in internships at this time. Additionally, exposure to platforms such as World Critical Dietetics conferences and involvement in advocacy groups within the national dietetic organization should be considered.

\section{Conclusions and Relevance to Practice}

This paper actively questions, challenges and counters the biases and assumptions built into the field of dietetics. As advocates, we add our voices to better train and sustain a profession that fully reflect the diverse Canadian society. We have attempted to highlight current recommendations from the literature and urge dietetic practitioners, leaders and educators to recognize barriers to success for racialized individuals in the profession. Promoting racial diversity in dietetics is everyone's responsibility and is in the best interest of the populations we serve. In order for us to see a paradigm shift, it is essential that we all commit to doing better. This will help build a new foundation for the next generation of dietetic practitioners, leaders and educators to create a more integrated practice that our current demographic needs. The first step is to acknowledge that racial diversity is lacking in dietetics and that systemic racism continues to exist in our 
profession and in Canada (based on the majority of the authors' experiences). By coming together, engaging in respectable and sustained conversations and remaining united, we can overcome racial discrimination in our field. The hope is to build a brighter future for the profession that is filled with equity and fairness for all.

\section{Acknowledgements}

Disclosure: The authors declare no conflict of interest.

Author contributions: AM and ATB conceptualized this paper. $A M$ wrote the manuscript and $A T B$ supervised this project. MR, AB, JL, FA, AMB, TP and VP edited the manuscript for intellect content. All authors reviewed and approved this manuscript.

Other contributors: All authors would like to thank Kehinde Sangill MPH, RD for her valuable feedback during the review of this paper.

\section{References}

Brady, J. (2018). Toward a critical, feminist sociology of expertise. Journal of Professions and Organization, 5(2), 123138. https://doi.org/10.1093/jpo/joy004

Brady, J., \& Gingras, J. (2019). Critical dietetics: Axiological foundations. In J. Coveney \& S. Booth (Eds.), Critical dietetics and critical nutrition studies (pp. 15-32). Springer. https://doi.org/10.1007/978-3-030-03/13-8_2

Burt, K. G., Delgado, K., Chen, M., \& Paul, R. (2019). Strategies and recommendations to increase diversity in dietetics. Journal of the Academy of Nutrition and Dietetics, 119(5), 733-738. https://doi.org/10.1016/j.jand.2018.04.008

Canadian Institute for Health Information. (1996-2020). Health workforce. Canada's Health Care Providers, 2015 to 2019 Data Tables. https://www.cihi.ca/en/health-workforce

Commission on Dietetic Registration [CDR]. (2020, August 10). Registered dietitian (RD) and registered dietitian nutritionist (RDN) by demographics. https://www.cdrnet. org/registry-statistics?id=3982\&action $\times$ m=ByDemograph ics

Council of Ontario Universities. (2013, June). Integrating Clinical Education into Ontario's Changing Health Care System. https://cou.ca/wp-content/uploads/2015/05/IntegratingClinical-Education-into-the-Changing-Healthcare-System. pdf

Government of Canada. (2019, July 25). Social determinants of health and health inequalities. https://www.canada.ca/ en/public-health/services/health-promotion/populationhealth/what-determines-health.html

Hall, W. J., Chapman, M. V., Lee, K. M., Merino, Y. M., Thomas, T. W., Payne, B. K., Eng, E., Day, S. H., \& Coyne-Beasley, T. (20I5). Implicit racial/ethnic bias among health care professionals and its influence on health care outcomes: A systematic review. American Journal of Public Health, 105 (12), e60-e76. https://dx.doi.org/10.2105\%2FAJPH.2015.302903

Huycke, P., Ingribelli, J., \& Rysdale, L. (2017). Aboriginal cultural competency in dietetics: A national survey of Canadian registered dietitians. Canadian Journal of Dietetic Practice and Research, 78(4), 172-176. https://doi.org//0.3148/ cjdpr-2017-009

Mattocks, K., \& Briscoe-Palmer, S. (2016). Diversity, inclusion and doctoral study: Challenges facing minority phd students in the United Kingdom. European Political Science, 15, 476492. https://doi.org/10.1057/s41304-016-007I-x

Mosby, I. (2013). Administering colonial science: Nutrition research and human biomedical experimentation in Aboriginal communities and residential schools, 1942-1952. Histoire sociale/Social History, 46(91), 145-172. https:// www.muse.jhu.edu/article/512043

National Collaborating Centre for Indigenous Health. (2012, January). The state of knowledge of Aboriginal health. A review of Aboriginal public health in Canada. https://www.nccih. ca/495/State_of_knowledge_of_Aboriginal_health__A_ review_of_Aboriginal_public_health_in_Canada. nccih?id=52

Riediger, N. D., Kingson, O., Mudryj, A., Farquhar, K. L., Spence, K. A., Vagianos, K., \& Suh, M. (2019). Diversity and equity in dietetics and undergraduate nutrition education in Manitoba. Canadian Journal of Dietetic Practice and Research, 80 (1), 44-46. https://doi.org/10.3148/cjdpr-2018-034

Polaschek, N.R. (1998). Cultural safety: a new concept in nursing people of different ethnicities. Journal of Advanced Nursing. 27(3), 452-457. http://doi.org/10.1046/j.13652648.1998.00547.x

Smedley, B. D., \& Mittman I. S. (20II). The diversity benefit: How does diversity among health professionals address public needs? In R. A. Williams (Ed.), Healthcare disparities at the crossroads with healthcare reform (pp. 167-193). Springer. https://doi.org/10.1007/978-1-4419-7136-4_II

Statistics Canada. (2017, October 25). Immigration and ethnocultural diversity: Key results from the 2016 census. https://www150.statcan.gc.ca/nl/daily-quotidien/171025/ dq $17 \mid 025$ b-eng.htm? indid $=\mid 4428-3$ \&indgeo $=0$

White, J. (2013). "Hearing the voices": African American nutrition educators speak about racism in dietetics. Journal of Critical Dietetics, I (3), 26-35. https://criticaldieteticsblog. files.wordpress.com/2013/04/journal-of-criticaldietetics-p26-35.pdf

White, J. H., \& Beto, J. A. (2013). Strategies for addressing the internship shortages and lack of ethnic diversity in dietetics. Journal of the Academy of Nutrition and Dietetics, 113(6), 771-775. https://doi.org/10.1016/j.jand.2013.03.012

White, J. (20/8). Barriers to becoming registered dietitians identified by African American students and practitioners. Journal of Clinical Dietetics, 4(I), 4I-46. https:// criticaldieteticsblog.files.wordpress.com/2018/07/4I-46white.pdf 


\section{Author Bios}

Anisha Mahajan is a full-time doctoral student at the University of Guelph and a Clinical Dietitian providing casual coverage for two hospital networks in Ontario, Canada. Anisha has worked as a registered dietitian for over 10 years in healthcare. She has completed her Master of Public Health (MPH) in Nutrition and Dietetics from the Dalla Lana School of Public Health at the University of Toronto, her dietetic internship from ARAMARK Canada Ltd. and her undergraduate degree from Ryerson University, Toronto in Nutrition and Food. Anisha has been involved in health promotion projects conducted in the region of Peel in Ontario such as the South Asian Adolescent Diabetes Awareness program (SAADAP). Anisha's current research interests includes understanding the impacts of dietary sugar intake in children and the importance of family-based health promotion programs in our communities. Anisha is also passionate to further her knowledge in promoting racial diversity in employment and educational settings and understanding the impact of food insecurity in Canada. Anisha identifies as a cis-gendered, middle-class female and is of South Asian descent. She was born and raised in Delhi, India before she moved to Ontario, Canada.

Dr. Ananya Tina Banerjee is an Assistant Professor at the Dalla Lana School of Public Health (DLPSH), University of Toronto. Her interdisciplinary research embeds a strong emphasis on community-based participatory research (CBPR), which is grounded in collaboration and partnership with South Asian communities at high risk for type 2 diabetes. Often, the research questions she pursues are community-defined problems in the context of the social determinants of health using mixed-methods. This allows for the innovative adaptation of existing resources; explores local knowledge and perceptions; empowers racialized people by considering them agents who can investigate their own situations. She created and developed the very first course on race and health in a school of public health in Canada. Ananya identifies as a cis-gendered, middle-class female and is of South Asian descent with ancestral roots from Kolkata, India.

Maria Ricupero is a practicing registered dietitian in Toronto, Ontario, who provides a weight inclusive approach for people living with chronic conditions including diabetes, heart disease, cancer and eating disorders. More recently, her practice interests have shifted to include trauma-informed care in dietetics. She has received trauma-informed training as a dietitian and certified yoga instructor. Maria is a white, cis-gendered, middle-class female.

Amanda Beales is a Registered Dietitian living in Toronto, Ontario and has practiced dietetics within a culturally diverse urban patient population for the past 12 years, primarily in adult inpatient care, in both England and Canada. Amanda's dietetic experience and interests include interprofessional collaboration and education, enteral nutrition, and gerontology and end of life care. Amanda has also volunteered with Dietitians of Canada's Gerontology Network since she was a student. Amanda is a white, cis-gendered middle-class female.

Jane Lac is a registered dietitian practicing at a Family Health Team in Etobicoke, Ontario. As a first generation post-secondary graduate, she obtained her Master of Public Health in Nutrition and Dietetics from the Dalla Lana School of Public Health at the University of Toronto, her dietetic internship from SickKids Hospital, and her undergraduate degrees from Ryerson University and McMaster University. Her continued interests include paediatric nutrition and women's health. Jane was born to East Asian parents who immigrated to Toronto, Canada as refugees escaping the Vietnam War. Jane's early exposure to food was through her mother's work in food production, and her father's almost 40 years of experience in the restaurant industry as a cook and previous Chinese (Szechuan style) restaurant owner. Jane identifies as a cis-gendered, middle-class female and is of East Asian descent.

Fatim Ajwani is a Registered Dietitian who has worked at the University Health Network Toronto Rehab Cardiac Rehabilitation and Secondary Prevention Program for the past 19 years. She provides both individual and group nutrition education to culturally diverse patients from the Greater Toronto Area who are living with cardiovascular disease and diabetes. She has been involved in several research and patient education projects including the translation and cultural adaptations of the Cardiac Rehab patient workbook and Cardiac College website, and the development of a food skills program for patients living with CVD. She is currently part of the Canadian Consortium of Neurodegeneration in Aging (CCNA) Diet and Lifestyle Team and is involved in the LEAD trial looking at the impact of diet and exercise on the prevention of cognitive impairment in older adults, as well as the development of the online Brain Health Pro program. Fatim has also 
been a long time volunteer for a community food bank that primarily serves the needs of newcomers to Toronto and has spent a summer volunteering at a hypertension and diabetes clinic in a community hospital in rural Zanzibar. Fatim comes from an East African background, is a cis-gendered, middle-class female.

Avantika Mathur-Balendra is a public health professional and a veterinary medicine student at the University of Melbourne. She graduated from the University of Waterloo with a BSc. in Health Studies and from McMaster University with a MSc. in Global Health. As a second generation immigrant whose family comes from Jaipur, India, she has always had a special interest in high-risk and marginalized populations. Her work has largely focused on developing and implementing health promotion and disease prevention initiatives, and she has worked on various youth- and community-driven programs in Peru, Costa Rica, Singapore, Canada and the US. With her experience in infectious and chronic disease prevention in humans, she is now turning her attention to veterinary public health initiatives and is involved with a number of animal health and wellness research projects. Avantika identifies as a cis-gendered, middle-class female. She has lived in India for a few years, and has spent most of her life in Ontario, Canada. She enjoys spending time with her dog and her husband, who is a first generation immigrant from Sri Lanka.

Twinkle Patel is a Registered Dietitian who has been actively engaged in the Seniors Care sector for over 10 years. Twinkle was exposed to dining services very early on in life, with her grandfather's restaurant business, serving over 300 customers at a time. Combining her love and understanding of food, she completed her graduation from Ryerson University in the Food and Nutrition Program. She has been working with Seasons Care for the last 10 years. Seasons Care's philosophy and approach to Food First was an immediate connection for Twinkle. She has worked in many different areas of dietetics including; Menu Planning for LTC, Clinical Dietitian, Home Care Dietitian, Recruitment and Training and Quality Improvement Programs and Clinical and Operational Leadership. Twinkle is also RAI-MDS certified. Twinkle identifies as a cis-gendered, middleclass female and is of South Asian descent. Twinkle was born and brought up in India, but has lived in various countries over the years, mainly within the UAE.

Vanita Pais is a Registered Dietitian (RD), Certified Diabetes Educator (CDE) and Certified insulin pump trainer (CPT). She has been practicing as a dietitian for over 20 years. She is a cis gender, middle class South Asian who started her practice in India and moved to Canada in 2000. In the last 15 years she has been working with the Endocrinology department at the Hospital for Sick Children. She also has held a Project Investigator position at the Sick Kids Research Institute for the past 10 years. Her areas of research interest are in Food Security in children with diabetes and developing videos and apps to teach carbohydrate counting and understand its impact on diabetes self-management and learning. She is particularly interested in improving care provided to patients and families and has led multiple Quality Improvement projects in the department. She was awarded the Banting and Best Diabetes Educator of the Year Award in 2019. She is passionate about helping people to improve their lifestyle and optimizing their diabetes management. She contributes her expertise in the community and volunteers as an Outreach and Learning Series presenter for Diabetes Canada. She likes cooking and trying out new recipes and enjoys long walks and hikes with her dog. 